\title{
Factores asociados al dolor cervical en estudiantes del nivel secundaria de una institución educativa estatal, Lima - Perú
}

\author{
Cristhian Santiago Bazan* 1,a; Jenny Rosado Ladera 2,b
}

RESUMEN

Objetivo: Determinar los factores asociados al dolor cervical en estudiantes de una institución educativa estatal en Lima, Perú.

Materiales y métodos: Estudio descriptivo, transversal, correlacional. Participaron 300 estudiantes de nivel secundario. Se confeccionó un cuestionario de 19 preguntas que fue validado por 11 expertos. Cada ítem fue evaluado (suficiencia, claridad, coherencia y relevancia), se aplicó el estadístico $V$ de Aiken $(0,91)$ y se midió la confiabilidad del instrumento mediante el alfa de Cronbach, con un resultado de 0,826 , que indica que el instrumento es altamente confiable para la asociación entre variables categóricas. Se empleó la prueba de Chi-Cuadrado para establecer las diferencias significativas $(p<0,05)$.

Resultados: Se encontró una relación estadísticamente significativa en cuanto al factor edad $(p=0,001)$ y el grado de escolaridad $(p=0,001)$ que presenta una correlación con el dolor cervical.

Conclusiones: El dolor en la región cervical es un problema de salud pública que no necesariamente se presenta en la edad adulta, sino también en edades tempranas, lo que se ha demostrado en la presente investigación. Si no es tratado a tiempo, conllevaría a una limitación funcional que afectaría la calidad de vida de la persona que lo padece.

Palabras clave: Dolor de cuello; Adolescente; Estudiantes; Enfermedades musculoesqueléticas (Fuente: DeCS BIREME).

\section{Factors associated with neck pain in high school students of a state educational institution, Lima - Peru}

\section{ABSTRACT}

Objective: To determine the factors associated with neck pain in students of a state educational institution in Lima, Peru.

Materials and methods: A descriptive, cross-sectional and correlational study was conducted with 300 high school students. A questionnaire consisting of 19 questions and validated by 11 experts was prepared. Each item was evaluated (sufficiency, clarity, coherence and significance) using the Aiken's V statistic $(0.91)$. The reliability of the instrument was determined with Cronbach's alpha (0.826), which indicated a high level of reliability of the instrument regarding the association among categorical variables. A chi-square test was used to establish the significant differences $(p<0.05)$. Results: A statistically significant relationship was found between cervical pain and factors such as age ( $p=0.001)$ and educational status $(p=0.001)$.

Conclusions: Neck pain is a public health problem. It does not necessarily occur in adulthood but also at early ages, which has been demonstrated in the present research. If not treated on time, it would lead to a functional limitation affecting the quality of life.

Keywords: Neck pain; Adolescent; Students; Musculoskeletal diseases (Source: MeSH NLM).

1. Centro de Rehabilitación Integral Física Funcional. Lima, Perú.

2. Universidad Católica Sedes Sapientiae, Facultad de Ciencias de la Salud, Tecnología Médica. Lima. Perú.

a. Doctor en Educación.

b. Bachiller en Tecnología Médica.

*Autor corresponsal 


\section{INTRODUCCIÓN}

El dolor cervical es un problema de salud pública que ha sido ampliamente estudiado en adultos, pero del que existe escasa información en el grupo de niños y adolescentes, que presentan numerosos factores de riesgo que contribuyen al desarrollo de este cuadro.

Los estudiantes de secundaria están expuestos a desarrollar dolor en la región cervical, que comienza a manifestarse de forma aguda, intermitente y, algunas veces, se irradia hacia la zona distal del miembro superior. Así mismo, puede presentarse con dolor, rigidez, disminución de la sensibilidad y parestesias. El dolor de cuello en la adolescencia es tan frecuente como en la edad adulta ${ }^{(1,2)}$.

El dolor musculoesquelético es común entre los adolescentes, pero se sabe poco acerca de los factores que influyen en que estos busquen atención médica para el problema. Los adolescentes que padecen dolor musculoesquelético y que consultan a un profesional de la salud son muy pocos ${ }^{(3)}$.

La cervicalgia es un importante problema de salud pública que ha sido ampliamente estudiado en adultos, pero no en niños y adolescentes, grupos que presentan numerosos factores de riesgo que contribuyen al desarrollo del cuadro. El aumento de las tensiones la columna cervical puede conducir a la degeneración cervical, junto con otras complicaciones del desarrollo, médicas, psicológicas y sociales ${ }^{(4)}$. Este padecimiento puede estar relacionado a dolor de cabeza ${ }^{(5)}$, migraña ${ }^{(6,7)}$, síntomas de ansiedad, depresión, agresividad ${ }^{(8)}$, estrés académico, actividad física, discapacidad cervical ${ }^{(9,10)}$ y problemas conductuales (11).

El padecer de dolor en la espalda durante la niñez o en la adolescencia, aumenta el riesgo de llegar a la cronicidad en la etapa adulta. El número de horas que los escolares permanecen en estado sedentario frente a la televisión o los videojuegos, el tipo de mochila que usan, y en la que transportan material escolar excesivamente pesado, representan factores de riesgo para generar dolores constantes en alguna zona del cuerpo, principalmente, en la columna vertebral (12). Además, muchas enfermedades musculoesqueléticas siguen un patrón a largo plazo de exacerbaciones y remisiones recurrentes, por lo que un predictor más consistente de un nuevo episodio es la experiencia de un episodio anterior ${ }^{(13)}$.

Los escolares adoptan patrones de postura al sentarse, cargar mochilas, en una marcha inadecuada, o bien al permanecer sentados por hasta 6 horas, con pequeños intervalos en pie. Todo esto puede ocasionar, alteraciones posturales, fatiga y dolores que, a la larga, podrían ser crónicos. La educación sanitaria es necesaria en esta población vulnerable ${ }^{(14,15)}$.

\section{MATERIALES Y MÉTODOS}

Diseño y población

Estudio descriptivo, correlacional, transversal y observacional. La población está conformada por los alumnos de la institución educativa Toribio Rodríguez de Mendoza. La muestra es de 300 estudiantes de secundaria, se obtuvo mediante un muestreo no probabilístico a criterio del autor, lo que se tendrá en cuenta al estudiar a cada uno de los elementos que componen la población.

\section{Variables y mediciones}

Se utilizó un cuestionario para la identificación de los factores posturales relacionados al dolor cervical en estudiantes del nivel secundario que consta de 19 preguntas y las variables: actitud postural en clase, actitud postural en el hogar, carga de peso de material educativo, transporte de material educativo, cuidado postural de la espalda, y educación sanitaria (conocimiento de la prevención del dolor cervical). El cuestionario fue validado por 11 expertos.

Las consideraciones de los jueces expertos al validar el instrumento fueron los siguientes indicadores de evaluación: suficiencia, claridad, coherencia y relevancia por cada pregunta. Seguidamente, se aplicó la técnica estadística $V$ de Aiken donde se obtuvo un puntaje de 0,91 , se tuvo en cuenta que el valor de esta técnica oscila desde 0 hasta 1 . Se considera el puntaje 1 como el máximo valor, lo que demuestra que existe entre los jueces expertos. Así mismo, el valor alcanzado por alfa de Crombach fue 0,826 , lo que indica que el instrumento es altamente confiable.

El método para recolectar la información fue la observación, que permite realizar un registro visual, consciente y dirigido que recolecta la información de las personas seleccionadas. Se elaboró una ficha de datos en las que consideró la información sociodemográfica (edad, sexo, estado civil) y presencia del dolor cervical.

\section{Análisis estadístico}

La base de datos hecha con la información obtenida se procesó con el paquete estadístico SPSS versión 22 para Windows 10 . Se utilizó el análisis descriptivo univariante y bivariante, en el cual la asociación entre variables categóricas se estableció a través de las pruebas de ChiCuadrado.

\section{RESULTADOS}

En la tabla 1 se observa una alta prevalencia de dolor cervical $(86,7 \%)$ en los estudiantes de educación secundaria de una I. E. de San Juan de Lurigancho. 
Factores asociados al dolor cervical en estudiantes del nivel secundaria de una institución educativa estatal, Lima - Perú

Tabla 1. Prevalencia de estudiantes de educación secundaria que padecen dolor cervical de una I. E. de San Juan de Lurigancho, 2018 $(n=300)$

\begin{tabular}{lccccccc} 
Dolor cervical & \multicolumn{2}{c}{ Presente } & \multicolumn{2}{c}{ Ausente } & & \\
& $\mathbf{n}$ & $\%$ & $\mathbf{n}$ & $\%$ & $\mathbf{n}$ & $\%$ \\
\hline Total & 260 & 86,7 & 40 & 13,3 & 300 & $100 \%$ \\
\hline
\end{tabular}

En la tabla 2 se presenta el cruce de las variables género y dolor cervical, se determinó que no existe asociación

estadísticamente significativa $(p=0,648)$ entre las variables mencionadas.

Tabla 2. Dolor cervical y género en los estudiantes de educación secundaria de una I. E. de San Juan de Lurigancho, $2018(\mathrm{n}=300)$

\begin{tabular}{|c|c|c|c|c|c|c|}
\hline \multirow[t]{3}{*}{ Género } & \multicolumn{4}{|c|}{ Dolor cervical } & \multicolumn{2}{|c|}{ Total } \\
\hline & \multicolumn{2}{|c|}{ Presente } & \multicolumn{2}{|c|}{ Ausente } & \multirow[b]{2}{*}{$\mathbf{n}$} & \multirow[b]{2}{*}{$\%$} \\
\hline & $\mathbf{n}$ & $\%$ & $\mathbf{n}$ & $\%$ & & \\
\hline Masculino & 146 & 85,9 & 24 & 14,1 & 170 & 100,0 \\
\hline Femenino & 114 & 87,7 & 16 & 12,3 & 130 & 100,0 \\
\hline Total & 260 & 86,7 & 40 & 13,3 & 300 & $100 \%$ \\
\hline Chi-cuadrado de Pearson $=0,209$ & g.l. $=1$ & $p=$ & & & & \\
\hline${ }^{*} p<0,05$ & & & & & & \\
\hline
\end{tabular}

En la tabla 3 se presentan las características sociodemográficas en los alumnos de educación secundaria. El género masculino fue el más frecuente
$(56,7 \%)$ y la edad promedio fue 14 años. Los participantes estaban distribuidos de manera homogénea (20\%), por cada uno de los años de estudio evaluados.

Tabla 3. Características sociodemográficas en los estudiantes de educación secundaria de una I. E. de San Juan de Lurigancho, 2018 $(n=300)$

\begin{tabular}{|ccc|}
\hline Características & Frecuencia & Porcentaje \\
\hline Género & & \\
Masculino & 170 & 170 \\
Femenino & 130 & 130 \\
Edad (años) & & $14 \pm 1,4^{*}$ \\
Año de estudio & & \\
Primero & 60 & 20,0 \\
Segundo & 60 & 20,0 \\
Tercero & 60 & 20,0 \\
Cuarto & 60 & 20,0 \\
Quinto & 60 & 20,0 \\
\hline
\end{tabular}

\footnotetext{
* Promedio \pm D.E.
} 
En la tabla 4 se presenta el cruce de las variables año de estudio y dolor cervical. Se determinó una asociación estadísticamente significativa $(\mathrm{p}=0,000)$ entre las variables mencionadas; es decir, el año de estudios está asociado al dolor cervical. Esta tendencia encontrada se puede apreciar en las diferencias de los porcentajes asociados según año de estudios: los estudiantes de segundo y cuarto año tienen los mayores porcentajes asociados a dolor cervical $(96,7 \%)$, y le sigue en importancia quinto año (90\%).

Tabla 4. Dolor cervical y año de estudio en los estudiantes de educación secundaria de una I. E. de San Juan de Lurigancho, 2018

\begin{tabular}{|c|c|c|c|c|c|c|}
\hline \multirow[t]{3}{*}{ Género } & \multicolumn{4}{|c|}{ Dolor cervical } & \multicolumn{2}{|c|}{ Total } \\
\hline & \multicolumn{2}{|c|}{ Presente } & \multicolumn{2}{|c|}{ Ausente } & \multirow[b]{2}{*}{$\mathbf{n}$} & \multirow[b]{2}{*}{$\%$} \\
\hline & n & $\%$ & $\mathbf{n}$ & $\%$ & & \\
\hline Primero & 46 & 76,7 & 14 & 23,3 & 60 & 100,0 \\
\hline Segundo & 58 & 96,7 & 2 & 3,3 & 60 & 100,0 \\
\hline Tercero & 44 & 73,3 & 16 & 26,7 & 60 & 100,0 \\
\hline Cuarto & 58 & 96,7 & 2 & 3,3 & 60 & 100,0 \\
\hline Quinto & 54 & 90,0 & 6 & 10,0 & 70 & 100,0 \\
\hline Total & 260 & 86,7 & 40 & 13,3 & 300 & $100 \%$ \\
\hline \multicolumn{5}{|c|}{$\begin{array}{l}\text { Chi-cuadrado de Pearson }=25,385 \text { g.l. }=4 \quad p=0,000^{*} \\
* \quad p<0,05\end{array}$} & & \\
\hline
\end{tabular}

\section{DISCUSIÓN}

La finalidad de la investigación es determinar los factores asociados al dolor cervical presentes en los alumnos de educación secundaria de la institución educativa Toribio Rodríguez de Mendoza a padecer algún tipo de discapacidad física a lo largo de su vida académica, ya que este problema es de interés de salud pública.

El dolor cervical en el estudiante de secundaria conlleva a la adopción de posturas compensatorias, lo que ocasiona una desalineación de los ejes corporales y, por ende, el acortamiento y elongación de las partes blandas de la región cervical (musculatura), lo que va a limitar los movimientos del estudiante en sus actividades diarias, y podría resultar en una discapacidad física.

En nuestro medio existe muy poca información sobre el dolor cervical en estudiantes de nivel secundario, por lo que es de suma importancia obtener datos epidemiológicos de esta situación, ya que este grupo es vulnerable a presentar episodios de dolor cervical a corta edad.

La investigación se realizó con una muestra de 300 estudiantes. Se observa una alta prevalencia de adolescentes que padecen dolor en la región cervical. Estudios similares a este trabajo como los de Zhou L (1),
Meziat-Filho $\mathrm{N}^{(2)}$, Fares J (4), Weber SA ${ }^{(5)}$, Canté XC (12), Scarabottolo CC (16), Shan Z (17), Shan Z (18), Briggs ${ }^{(19)}$ refieren la importancia de la variable dolor cervical, que es un padecimiento que puede ocasionar una limitación funcional. Los alumnos de educación secundaria no son ajenos a padecerlo por su corta edad, si no que el cuadro podría estar relacionado a factores (ambientales y de estilo de vida) a los que están expuestos ${ }^{(20)}$.

Los datos epidemiológicos encontrados en la presente investigación hacen mención a que existe una alta prevalencia de dolor cervical en los estudiantes de la institución educativa. Autores como Scarabottolo $\mathrm{CC}^{(16)}$ reportan también una alta prevalencia de este padecimiento. Si bien este problema es frecuente es personas adultas, los niños y adolescentes no son ajenos a padecer este problema musculoesquelético.

Por otro lado, el sexo femenino es el que presenta un mayor porcentaje de dolor cervical. Estudios similares como Fares J (4), Shanz Z (17), Shan Z (18), Dianat I (21), Ruivo $\mathrm{RM}^{(22)}$ concuerdan con el nuestro en que las mujeres presentan mayor probabilidad que los varones en padecer dolor de cuello. Es necesario promover programas de intervención médica en este grupo afectado para poder mejorar el bienestar de los estudiantes.

En cuanto a las variables estadísticamente significativas, 
nuestro estudio muestra similitudes con las investigaciones de Fares J (4), Canté XC ${ }^{(12)}$, al relacionar el dolor cervical y la edad de los estudiantes; y con Shanz $Z^{(17)}$ y Shan $Z{ }^{(18)}$, cuando se relaciona el dolor cervical al grado de escolaridad.

Observamos que, cuanto más asciende el grado académico, los estudiantes asumen mayor responsabilidad en su formación y se enfrentan a situaciones de alta demanda, lo que les permite ser personas de bien. Así mismo, aumenta la capacidad de adaptación a los estresores internos y externos, de acuerdo a la etapa evolutiva alcanzada.

Estos cambios dependen del nivel de actividad física del estudiante ${ }^{(19)}$ y el tipo de estilo de vida que llevan cada uno ellos de forma independiente, cuadros adaptativos, ansiosos, conductuales y emocionales ${ }^{(20)}$.

Las molestias musculoesqueléticas, hoy en día, se presentan con mayor frecuencia en escolares ${ }^{(21,23)}$; es necesario investigar este tipo de afección en etapas tempranas de la vida porque el dolor podría hacerse crónico en la adultez ${ }^{(24-26)}$.

Por otro lado, no se encontró una relación estadísticamente significativa entre actitud postural en clase, actitud postural en el hogar, carga y transporte de material educativo, cuidado postural de la espalda y educación sanitaria (conocimiento de la prevención del dolor cervical) con la presencia de dolor cervical en los alumnos de este estudio.

Como limitación de la investigación, señalaremos que el diseño de estudio es de tipo transversal, lo que no permite dar un seguimiento de la muestra ya que solo se puede recolectar información en un momento y tiempo únicos. El tipo de muestreo empleado es no probabilístico a criterio del autor, lo que no permitió extrapolar los datos a otras poblaciones de similares características. Se escogió este criterio dada la accesibilidad y proximidad de los sujetos a investigar. Además, no se consideró el nivel de discapacidad que conlleva el dolor cervical y los estados emocionales. Myrtveit SM (11), hace referencia a que los factores conductuales y emocionales se asocian con el riesgo de dolor en el cuello. Así mismo, existe escasa información acerca de este tema en edades tempranas.

El aporte al conocimiento científico se basa en la identificación de la frecuencia de dolor cervical y los hábitos posturales de los estudiantes de secundaria. Esta investigación ayudará a reforzar conceptos acerca de este padecimiento y conocer uno de los principales problemas que presentan los estudiantes de educación secundaria a nivel nacional ya que la información epidemiológica de este cuadro es escasa.

En conclusión, los factores asociados encontrados, si bien no pueden modificarse, sí podrían prevenirse, mediante la gestión de intervenciones en el ámbito escolar dirigidas a fomentar estilos de vida saludables en los adolescentes, así como la intervención especializada temprana para poder mitigar los problemas que conlleva este padecimiento.

\section{REFERENCIAS BIBLIOGRÁFICAS}

1. Zhou L, Huang YY, Chen DY, Zhang D, Luo QS, Wang Y, et al. Correlation between both neck/shoulder and low back pain and daily behavioral habits among middle school students in Shenzhen. Zhonghua Liu Xing Bing Xue Za Zhi. 2018; 39(4): 469-73.

2. Meziat-Filho N, Azevedo ESG, Coutinho ES, Mendonça R, Santos V. Association between home posture habits and neck pain in high school adolescents. J Back Musculoskelet Rehabil. 2017; 30(3): 467-75.

3. Paananen MV, Taimela SP, Tammelin TH, Kantomaa MT, Ebeling $\mathrm{HE}$, Taanila AM, et al. Factors related to seeking health care among adolescents with musculoskeletal pain. Pain. 2011; 152(4): 896-903.

4. Fares J, Fares MY, Fares Y. Musculoskeletal neck pain in children and adolescents: risk factors and complications. Surg Neurol Int. 2017; 8: 72.

5. Weber Hellstenius SA. Recurrent neck pain and headaches in preadolescents associated with mechanical dysfunction of the cervical spine: a cross-sectional observational study with 131 students. J Manipulative Physiol Ther. 2009; 32(8): 625-34.

6. Carvalho GF, Chaves TC, Gonçalves MC, Florencio LL, Braz CA, Dach $F$, et al. Comparison between neck pain disability and cervical range of motion in patients with episodic and chronic migraine: a cross-sectional study. J Manipulative Physiol Ther. 2014; 37(9): 641-6.

7. Pradhan S, Choudhury SS. Clinical characterization of neck pain in migraine. Neurol India. 2018; 66(2): 377-84.

8. Rees CS, Smith AJ, O'Sullivan PB, Kendall GE, Straker LM. Back and neck pain are related to mental health problems in adolescence. BMC Public Health. 2011; 11: 382.

9. Cillero Rodrigo A, Mayoral Gómez G, Mesa Fuentes S. Estudio piloto correlacional sobres las variables influyentes en la discapacidad cervical en estudiantes de la Universidad de Alcalá. Rev Univ Inf Inv Fisioter. 2014; 43(1): 30-7.

10. Young IA, Dunning J, Butts R, Cleland JA, Fernández-de-LasPeñas C. Psychometric properties of the Numeric Pain Rating Scale and Neck Disability Index in patients with cervicogenic headache. Cephalalgia. 2019 Jan; 39(1): 44-51.

11. Myrtveit SM, Sivertsen B, Skogen JC, Frostholm L, Stormark $K M$, Hysing M. Adolescent neck and shoulder pain-the association with depression, physical activity, screen-based activities, and use of health care services. J Adolesc Health. 2014 Sep; 55(3): 366-72.

12. Canté Cuevas XC, Kent Sulú MP, Vásquez Gutiérrez MG, Lara Severino RC. Factores posturales de riesgo para la salud en escolares de Ciudad del Carmen, Campeche. UNACAR Tecnociencia. 2010 Ene-Jun; 4(1): 1-15. 
13. Taylor JB, Goode AP, George SZ, Cook CE. Incidence and risk factors for first-time incident low back pain: a systematic review and meta-analysis. Spine J. 2014 Oct 01; 14(10): 2299319.

14. Ribeiro de Oliveira A, Rodrigues Scartoni F. Alteraciones posturales de alumnos de $5^{\mathrm{a}}$ y $6^{\mathrm{a}}$ series de la enseñanza fundamental. Fit Perf J. 2008 Ene-Feb; 7(1): 10-5.

15. Casas Sánchez AS, Patiño Segura MS. Prevalencia y factores asociados con el dolor de espalda y cuello en estudiantes universitarios. Rev Univ Ind Santander Salud. 2012; 44(2): 4555.

16. Scarabottolo CC, Pinto RZ, Oliveira CB, Zanuto EF, Cardoso JR, Christofaro DGD. Back and neck pain prevalence and their association with physical inactivity domains in adolescents. Eur Spine J. 2017 Sep; 26(9): 2274-80.

17. Shan Z, Deng G, Li J, Li Y, Zhang Y, Zhao Q. How schooling and lifestyle factors effect neck and shoulder pain? A crosssectional survey of adolescents in China. Spine (Phila Pa 1976). 2014 Feb; 39(4): E276-83.

18. Shan Z, Deng G, Li J, Li Y, Zhang Y, Zhao Q. Correlational analysis of neck/shoulder pain and low back pain with the use of digital products, physical activity and psychological status among adolescents in Shanghai. PLoS One. 2013 Oct 11; 8(10): e78109.

19. Briggs AM, Straker LM, Bear NL, Smith AJ. Neck/shoulder pain in adolescents is not related to the level or nature of selfreported physical activity or type of sedentary activity in an Australian pregnancy cohort. BMC Musculoskelet Disord. 2009 Jul 20; $10: 87$.

20. Maturana A, Vargas A. El estrés escolar. Rev Med Clin Las Condes. 2015 Jan-Feb; 26(1): 34-41.

21. Dianat I, Sorkhi N, Pourhossein A, Alipour A, AsghariJafarabadi $M$. Neck, shoulder and low back pain in secondary schoolchildren in relation to schoolbag carriage: should the recommended weight limits be gender-specific?. Appl Ergon. 2014 May; 45(3): 437-42.

22. Ruivo RM, Pezarat-Correia P, Carita Al. Cervical and shoulder postural assessment of adolescents between 15 and 17 years old and association with upper quadrant pain. Braz J Phys Ther. 2014 Jul-Aug; 18(4): 364-71.

23. Andias R, Silva AG. A systematic review with meta-analysis on functional changes associated with neck pain in adolescents. Musculoskeletal Care. 2019 Mar; 17(1): 23-36.

24. Kamper SJ, Henschke N, Hestbaek L, Dunn KM, Williams CM. Musculoskeletal pain in children and adolescents. Braz J Phys Ther. 2016 May-Jun; 20(3): 275-84.

25. De Vitta A, Trize DM, Fiorelli A, Carnaz L, Souza De Conti MH, Penteado Simeão SFA. Neck/shoulders pain and its relation to the use of tv/computer/videogame and physical activity in school students from Bauru. Fisioter Mov. 2014 Jan-Mar; 27(1): 111-8.

26. Ståhl MK, El-Metwally AA, Mikkelsson MK, Salminen JJ, Pulkkinen LR, Rose RJ, et al. Genetic and environmental influences on non-specific neck pain in early adolescence: a classical twin study. Eur J Pain. 2013 Jul; 17(6): 791-8.

Fuentes de financiamiento:

Este artículo ha sido financiado por los autores.

Conflictos de interés:

Los autores declaran no tener conflictos de interés.

\section{Correspondencia:}

Cristhian Santiago Bazán

Dirección: Jr. Tiahuanaco 1493 Urb. Zárate S.J.L. Lima, Perú.

Teléfono: 941703104

Correo electrónico: cristhiansantiagob@gmail.com

\section{Recibido: 19 de setiembre de 2018 .}

Evaluado: 17 de octubre de 2018. Aprobado: 13 de noviembre de 2018.

(๔) La revista. Publicado por Universidad de San Martín de Porres, Perú. (c) Br Licencia de Creative Commons Artículo en acceso abierto bajo términos de Licencia Creative Commons Atribución 4.0 Internacional. (http://creativecommons.org/licenses/by/4.0/)

ORCID iDs

Cristhian Santiago Bazan Jenny Mariela Rosado Ladera https://orcid.org/0000-0001-9073-4785 https://orcid.org/0000-0003-3018-398X 